\title{
Angiomyolipoma of the Jejunum Mimicking Metastatic Disease in a Patient with Colonic Adenocarcinoma
}

\author{
Spyridon Miliaras ${ }^{1}$, Dimosthenis Miliaras ${ }^{2}$ \\ ${ }^{1}$ Surgical Department, Papageorgiou Hospital, Medical School, Aristotle University, Thessaloniki, Greece \\ ${ }^{2}$ Laboratory of Histology and Embryology, Medical School, Aristotle University, Thessaloniki, Greece \\ E-mail:dmiliara@med.auth.gr \\ Received July 27, 2010; revised January 5, 2011; accepted March 9, 2011
}

\begin{abstract}
Angiomyolipoma is a benign tumor most commonly arising in the kidney. Very few cases have been reported to be located in the small intestine. Here we report the first case located in the jejunum in a patient who was diagnosed with a colonic adenocarcinoma. In the preoperative evaluation this benign lesion was thought it might represent a metastatic nodule.
\end{abstract}

Keywords: Angiomyolipoma, Colon Adenocarcinoma, Jejunum

\section{Introduction}

Angiomyolipoma (AML) is a benign neoplasm composed of blood vessels, smooth muscle cells, and mature fat cells [1]. AML most commonly arises in the kidney. Other locations include the liver (most frequent extrarenal site) [2], the uterus[3], the lungs [4], the skin [5], the subgaleal space [6], the anterior mediastinum [7], the urinary bladder [8], and mucosal membranes such as the lips [9] and the nasal cavity [10]. Very few cases have been reported in the gastrointestinal tract, including nine cases arising from the colon [11-19], and four cases arising from the small bowel [20-23]. Two of the latter cases were located in the duodenum [20,21], and the other two in the ileum $[22,23]$. In this paper we report a fifth case of AML of the small intestine, this time located at the jejunum. The benign small intestinal tumor was masquerading a metastatic nodule in the preoperative imaging evaluation, since our patient also presented a tumor in the ileocecal region.

\section{Case Report}

An 80 years old male was urgently admitted to our surgical department with abdominal pain, vomiting and abdominal distension. On clinical examination the patient was afebrile and the abdomen was moderately distended, with mild tenderness on palpation of the right iliac fossa, but no signs of peritonism. Bowel sounds were reduced and digital examination of the rectum was normal. Plain abdominal films confirmed small bowel obstruction and full blood count and biochemistry were unremarkable. Conservative treatment was initiated, including intravenous fluids, nasogastric suction and monitoring of the vital organs. After three days of conservative treatment, although there was no clinical deterioration, the obstructive ileus was not resolved and a colonoscopy was performed, to further investigate the etiology of obstruction, which revealed an obstructive tumor of the ileocecal valve. A preoperative $\mathrm{CT}$ scan of the abdomen performed the next day for staging purposes showed regional lymphadenopathy of the mesentery of the ileocecal region, but no hepatic metastases. However, a soft tissue mass was found in the proximal jejunum with most probable diagnosis to be metastatic implant (Figure 1(a)).

In order to resolve the persisting ileus, an exploratory laparotomy was decided and performed through a high suprasubumbilical incision. A large obstructive tumor of the ileocecal valve with regional lymphadenopathy were detected with no signs of hepatic or other metastatic disease. However, a round, well circumscribed tumor of the antimesenteric border of the proximal jejunum was detected (Figure 1(b)), which was excised in healthy tissues macroscopically through a limited small bowel enterectomy. A typical right hemicolectomy was then performed with ileocolic anastomosis to the transverse colon. Following an uneventful postoperative period, and after gradual mobilization and feeding, the patient was discharged home on the seventh postoperative with instructions for regular follow-up and referral to the medical 


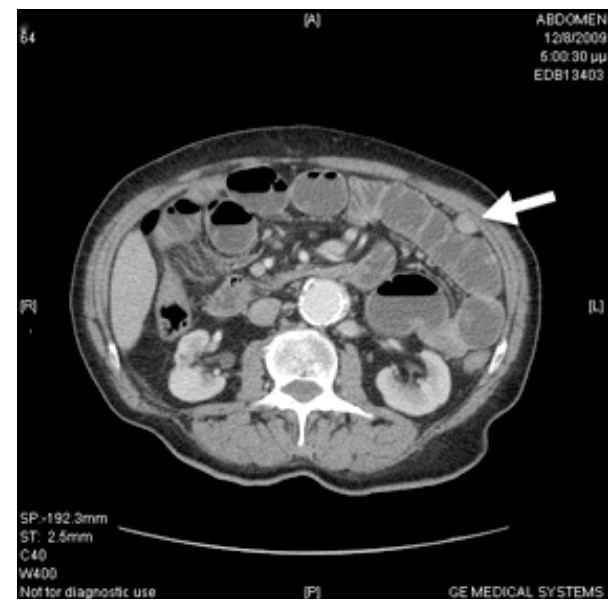

(a)

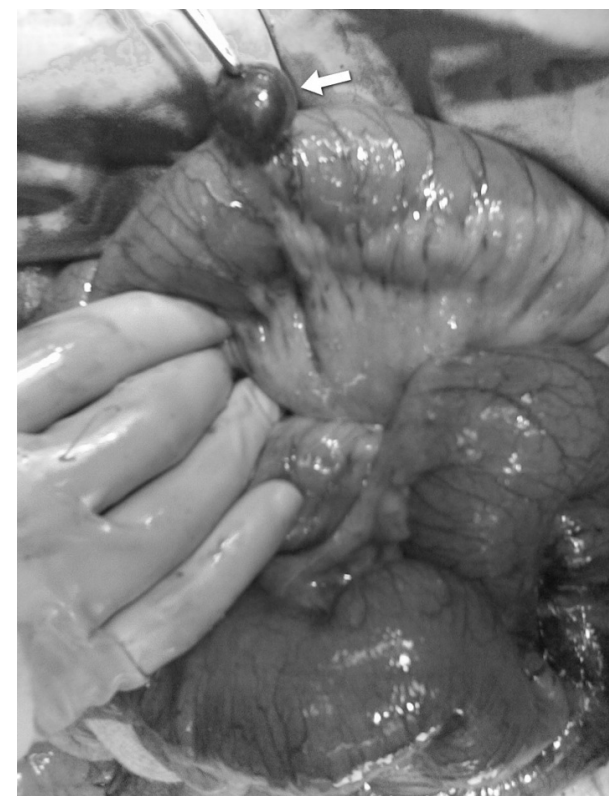

(b)

Figure 1. (a) CT scan of the abdominal area showing a nodule attached to the jejunum (arrow); (b) The surgical specimen of the nodule of the jejunum as found at the operation theater (arrow).

oncology department for the colon cancer. The patient refused any further treatment, but still today, 12 months after operation, is alive and well.

\subsection{Pathological Findings}

The hemicolectomy specimen consisted of a segment of the terminal segment of ileum $22 \mathrm{~cm}$ in length, the ileocecal valve, the cecum along with the appendix, and a segment of the ascending colon $16 \mathrm{~cm}$ in length. A large exophytic and invasive tumor, $4 \mathrm{~cm}$ in diameter was found in the ascending colon, close to the ileocecal valve. 20 lymph nodes, $0.3-1.2 \mathrm{~cm}$ in diameter were removed from the surrounding pericolic fat. On microscopy this tumor proved to be a well-differentiated adenocarcinoma of the large intestine, invading the whole thickness of the intestinal wall, and extending to the pericolic fat. One of the removed lymph nodes presented metastasis from the aforementioned adenocarinoma. The tumor of the jejunum was reddish, and soft, $2 \mathrm{~cm}$ in diameter. It was composed by spindle cells arranged in fascicles, vacuolated (fat) cells and many blood vessels (Figure 2(a)). No significant nuclear atypia, mitotic activity, necroses or increased cellularity were detected. In order to sustain a definitive diagnosis, immunohistochemical evaluation followed.

Immunohistochemistry was performed on formalinfixed, paraffin-embedded tissue sections, $3-\mu \mathrm{m}$ in thickness. High temperature antigen unmasking in electric pressure cooker, and TrilogyTM solution (Cell Marque, Rocklin, CA, USA) pretreatment preceded the main procedure. A standardised automated (Nexes, Ventana, Tuscon, AZ, USA), streptavidin-biotin method (I-VIEW Paraffin DAB, Ventana) followed the application of the monoclonal antibodies. The latter were directed against HMB-45 (dilution 1:50, Dako, Glostrup, Denmark), Melan-A (dilution 1:50, Dako), a-smooth muscle actin (SMA, dilution 1:40, Novocastra, Newcastle, UK), Desmin (dilution 1:100, Novocastra), S-100 (dilution 1:300, Dako), CD117 (dilution 1:50, Dako), and CD34 (dilution 1:50, Dako). The tumor cells were positive to HMB-45 (Figure 2(b)), Melan-A, SMA (Figure 2(c)), and desmin, while they were negative to CD117, and CD34 (except of the endothelial cells of the vessels, Figure 2(d)). Our findings in total were consistent with a diagnosis of angiomyolipoma.

\section{Discussion}

Traditionally, AML was considered as hamartoma. However, AML today is regarded as a true neoplasm, since it has been found to present clonal nature [24]. Moreover, AML is thought to arise from the perivascular epithelioid cell (PEC), which has certain morphologic and immunohistochemical characteristics, even though no known normal cell counterpart has been found until now [1]. Immunohistochemically, PEC expresses myogenic and melanocytic markers, such as HMB-45, HMSA-1, MelanA/Mart1, microophtalmia transcription factor (Mitf), actin and, less commonly, desmin. Other (rare) tumors which arise from PEC include clear-cell "sugar" tumor of the lung and extrapulmonary sites, lymphangioleiomyomatosis, clear-cell myomelanocytic tumor of the falciform ligament/ligamentum teres, and rare clear-cell tumors of other anatomical sites. In addition, some PEComas are related to the tuberous sclerosis 


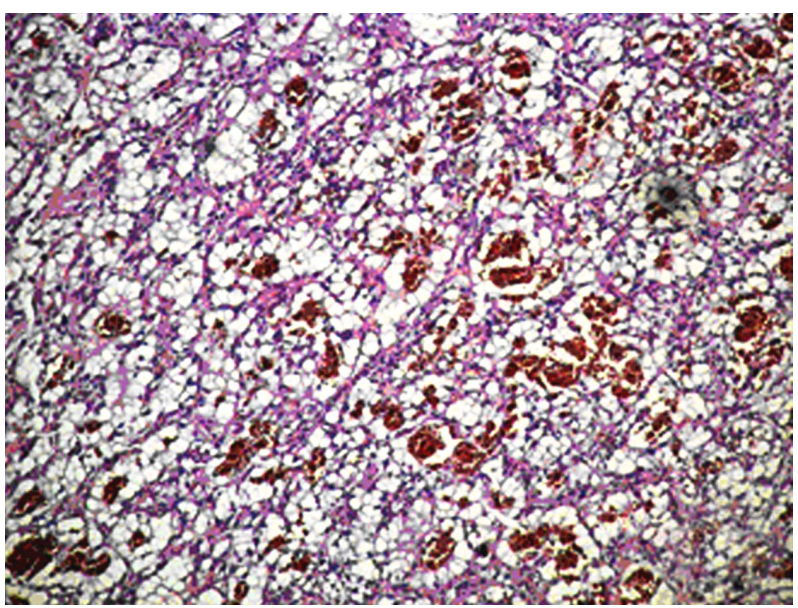

(a)

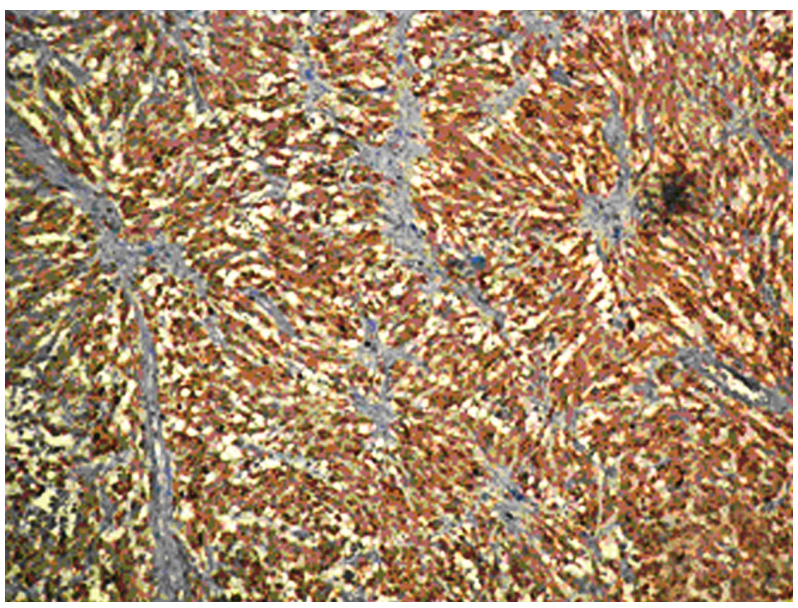

(c)

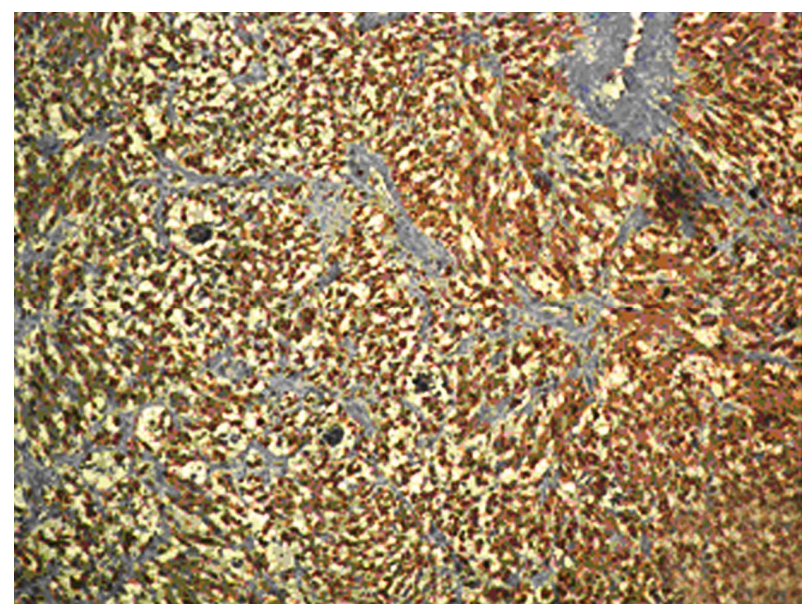

(b)

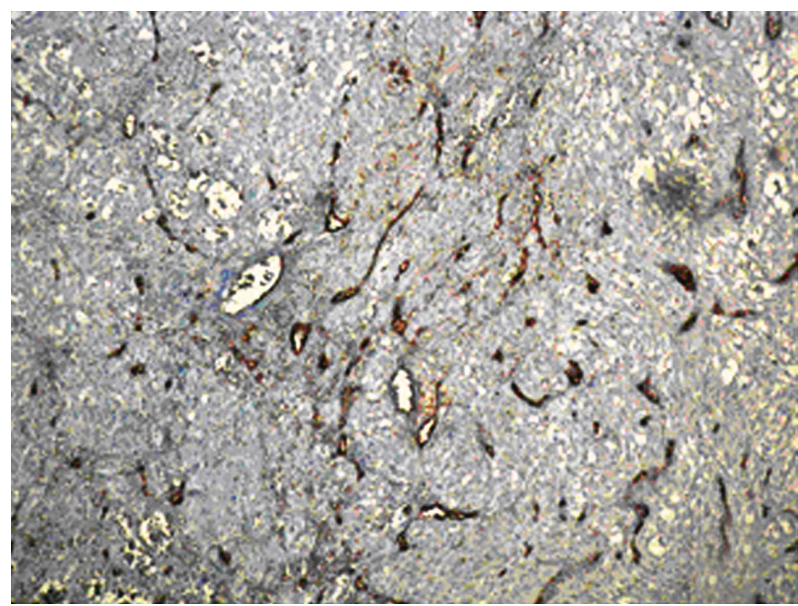

(d)

Figure 2. (a) The tumor is composed of fascicles of spindle cells, interspersed with groups of vacuolated fat cells and numerous blood vessels (H\&E, X100); (b) The spindle cells of the lesion are strongly positive to HMB-45 (DAB, Hematoxylin, X100); (c) The spindle cells of the lesion are strongly positive to smooth muscle actin (DAB, Hematoxylin, X100); (d) Only blood vessels show positive reaction to CD34 (DAB, Hematoxylin, X100).

complex (TSC), an autosomal dominant genetic disease due to losses of TSC1 (9q34) or TSC2 (16p13.3) genes $[25,26]$. This syndrome is characterized by mental retardation, seizures and cellular proliferations (AMLs, subependymal giant cell tumors, cutaneous angiofibromas, cardiac rabdomyomas, lymphangioleiomyomatosis, pulmonary multifocal micronodular hyperplasia). Similar alterations of the TSC genes have been demonstrated in a significant number of PEComas, both occurring within the TSC and in sporadic cases.

Sporadic AMLs occur in older patients, in the fourth to sixth decades of life, with a female predominance; they are single, unilateral and larger than those associated with TSC [27]. Classic AML contains more than one cell type; if a particular cell type predominates, AML is consequently named (lipoma-like AML or leiomyoma-like AML). AMLs arising in the GI tract are extremely uncommon and usually present with melena, anemia, diarrhea, abdominal pain, and may even be clinically asymptomatic [11-23]. Radiological diagnosis of extrarenal AMLs is difficult because of the rarity of the condition. Even though four other angiomyolipoma cases have been reported to affect small intestine, our case is unique, since to our knowledge is the first to involve jejunum. In addition, in our case the intestinal nodule was thought to represent metastatic disease in the pre-operative evaluation, because our patient was having a colonic tumor as well. So, it represented a critical differential diagnostic problem in terms of severity, and staging of his primary disease. Moreover, we consider ours to be a genuine AML case, since it is both HMB-45, and Melan-A positive. At least two cases of small intestinal AML reported in the literature [8,9] were HMB-45 negative (Table 1), when HMB-45 positive immureaction 
Table 1. Angiomyolipomas of the small intestine reported in the literature: Immunohistochemical reaction to various antibodies.

\begin{tabular}{lcccccccc}
\hline \multicolumn{1}{c}{ Author } & SMA & Desm & KIT & CD34 & Melan-A & HMB-45 & S-100 & Vim \\
\hline De Padua et al. & + & NA & NA & NA & NA & + & NA & NA \\
Toye and Czarnecki & + & NA & NA & NA & NA & - & NA \\
Lee et al. & + & + & - & + & NA & - & NA & NA \\
Lin et al. & + & + & + & + & + & + & NA & - \\
Miliaras and Miliaras & + & - & - & - & + & + & NA \\
\hline
\end{tabular}

$\mathrm{SMA}=$ smooth muscle actin, Desm $=$ desmin, Vim $=$ vimentin, $\mathrm{NA}=$ not available

is currently considered as a prerequisite for such a diagnosis $[1,14]$. In conclusion, intestinal AMLs are very rare, and may cause various symptoms or mimick other conditions as metastatic disease in our case. For this reason it is quite important to differentiate such a case from other mesenchymal small intestinal tumors, and especially from gastrointestinal stromal tumor, which is the most frequent tumor in this location and merits specialized targeted immunotherapy.

\section{References}

[1] G. Martignoni, M. Pea, D. Reghellin, G. Zamboni and F. Bonetti, "Pecomas: The Past, the Present and the Future," Virchows Archives, Vol. 452, No. 2, February 2008, pp. 119-132. doi:10.1007/s00428-007-0509-1

[2] P. J. Xu, Y. Shan, F. H. Yan, Y. Ji, Y. Ding and M. L. Zhou, "Epithelioid Angiomyolipoma of the Liver: Cross-Sectional Imaging Findings of 10 Immunohistochemically-Verified Cases," World Journal of Gastroenterology, Vol. 15, No. 36, September 2009, pp. 4576-4581. doi:10.3748/wjg. 15.4576

[3] A. P. Cil, A. Haberal, S. Hucumenoglu, E. E. Kovalak and M. Gunes, "Angiomyolipoma of the Uterus Associated with Tuberous Sclerosis: Case Report and Review of the Literature," Gynecologic Oncology, Vol. 94, No. 2, August 2004, pp. 593-596. doi:10.1016/j.ygyno.2004.05.015

[4] D. G. Jr. Guinee, D. S. Thornberry, N. Azumi, R. M. Przygodzki, M. N. Koss and W. D. Travis, "Unique Pulmonary Presentation of an Angiomyolipoma. Analysis of Clinical, Radiographic, and Histopathologic Features," American Journal of Surgical Pathology, Vol. 19, No. 4, April 1995, pp. 476-480.

[5] K. Singh, R. R. Pai, H. Kini and U. A. Kini, "Cutaneous Angiomyolipoma," Indian Journal of Pathology Microbiology, Vol. 52, No. 2, April-June 2009, pp. 242-243. doi:10.4103/0377-4929.48932

[6] A. Tamura, O. Ishikawa and Y. Miyachi, "Subgaleal Angiomyolipoma", Journal of Dermatology, Vol. 21, No. 7, July 1994, pp. 514-517.

[7] C. S. Knight, R. J. Cerfolio and T. S. Winokur, "Angio- myolipoma of the Anterior Mediastinum", Annals of Diagnostic Pathology, Vol. 12, No. 4, August 2008, pp. 293-295. doi:10.1016/j.anndiagpath.2006.12.007

[8] Y. Huan, R. W. Dillon and P. D. Unger, "Angiomyolipoma of the Bladder", Annals of Diagnostic Pathology, Vol. 6, No. 6, December 2002, pp. 378-380. doi:10.1053/adpa.2002.36655

[9] A. A. da Silva, R. Carlos, E. Contreras, O. P. de Almeida, M. A. Lopes and P. A. Vargas, "Angiomyolipoma of the Upper Lip: Case Report and Review of the Literature," Medicina Oral, Patología Oral y Cirugía Bucal, Vol. 12, No. 2, March 2007, pp. E101-104.

[10] K. Watanabe and T. Suzuki T, "Mucocutaneous Angiomyolipoma: A Report of 2 Cases Arising in the Nasal Cavity", Archives of Pathology and Laboratory Medicine, Vol. 123, No. 9, September 1999, pp. 789-792.

[11] K. Oishi, S. Fukuda, H. Sakimoto, T. Eto, M. Takahashi and T. Nishida, "Angiomyolipoma of the Colon: Report of a Case", Surgery Today, Vol. 39, No. 11, November 2009, pp. 998-1001. doi:10.1007/s00595-009-3973-1

[12] H. Cheng, M. D. Sitrin and A. Rani A, "Angiomyolipoma in the Colon", Gastrointestinal Endoscopy, Vol. 64, No. 3, September 2006, pp. 443-444. doi:10.1016/j.gie.2006.01.064

[13] A.I. Sharara and A. Tawil A, "Angiomyolipoma of the colon", Clinical Gastroenterology and Hepatology, vol. 3 , no.9, September 2005, p. A35. doi:10.1016/S1542-3565(05)00287-9

[14] J. Pelz, K. Weber, J. Göhl, A. Dimmler and W. Hohenberger, "Angiomyolipoma of the Colon - Case Report and Review of the Literature", Zeitschrift für Gastroenterologie, Vol. 41, No. 8, August 2003, pp. 715-718.

[15] J. S. Chen, L. J. Kuo, P. Y. Lin and C. R. Changchien, "Angiomyolipoma of the Colon: Report of a Case and Review of the Literature", Diseases of Colon and Rectum, Vol. 46, No. 4, April 2003, pp. 547-549. doi:10.1007/s10350-004-6598-x

[16] H. Maluf and Dieckgraefe B, "Angiomyolipoma of the Large Intestine: Report of a Case", Modern Pathology, Vol. 12, No.12, December 1999, pp. 1132-1136.

[17] C. Maesawa, G. Tamura, H. Sawada, S. Kamioki, Y. Nakajima and R. Satodate, "Angiomyolipoma Arising in the Colon", American Journal of Gastroenterology, Vol. 
91, No. 9, September 1996, pp. 1852-1854.

[18] R. Verzaro, S. Guadagni, A. Agnifili, I. Ibi, P. Gola, F. Gianfelice, D. Ranalletta, G. Carducci and P. Leocata, “A Case of Extrarenal Angiomyolipoma with Intestinal Localization", Minerva Chirurgica, Vol. 47, No. 15-16, August 1992, pp. 1317-1319.

[19] Y. Hikasa, T. Narabayashi, M. Yamamura, Y. Fukuda, N. Tanida, K. Tamura, T. Ohno, T. Shimoyama and Nishigami T, "Angiomyolipoma of the Colon: A New Entity in Colonic Polypoid Lesions", Gastroenterologia Japonica, Vol. 24, No. 4, August 1989, pp. 407-409.

[20] M. De Padua, N. Gupta, S.L. Broor and D. Govil, "Duodenal Angiomyolipoma: A Case Report," Indian Journal of Pathology and Microbiology, Vol. 50, No. 3, July 2007, pp. 568-569.

[21] L. R. Toye and L. A. Czarnecki, "CT of Duodenal Angiomyolipoma," American Journal of Roentgenology, Vol. 178, No. 1, January 2002, p. 92.

[22] C. H. Lee, J. H. Kim, D. H. Yang, Y. Hwang, M. J. Kang, Y. K. Kim and M. R. Lee, "Ileal Angiomyolipoma Manifested by Small Intestinal Intussusception," World Journal of Gastroenterology, Vol. 15, No. 11, March 2009, pp. 1398-1400. doi:10.3748/wjg.15.1398

[23] C. Y. Lin, H. Y. Chen, S. C. Jwo and S. C. Chan, "Ileal Angiomyolipoma as an Unusual Cause of Small-Intestinal Intussusception," Journal of Gastroenterology, Vol. 40, No. 2, February 2005, pp. 200-203. doi:10.1007/s00535-004-1527-2

[24] L. Cheng, J. Gu, J. N. Eble, D. G. Bostwick, C. Younger, G. T. MacLennan, F. W. Abdul-Karim, W. A. Geary, M.
O. Koch, S. Zhang and T. M. Ulbright, "Molecular Genetic Evidence for Different Clonal Origin of Components of Human Renal Angiomyolipomas," American Journal of Surgical Pathology, Vol. 25, No. 10, October 2001, pp. 1231-1236. doi:10.1097/00000478-200110000-00002

[25] M. van Slegtenhorst, R. de Hoogt, C. Hermans, M. Nellist, B. Janssen, S. Verhoef, D. Lindhout, A. van den Ouweland, D. Halley, J. Young, M. Burley, S. Jeremiah, K. Woodward, J. Nahmias, M. Fox, R. Ekong, J. Osborne, J. Wolfe, S. Povey, R. G. Snell, J. P. Cheadle, A. C. Jones, M. Tachataki, D. Ravine, J. R. Sampson, M. P. Reeve, P. Richardson, F. Wilmer, C. Munro, T. L. Hawkins, T. Sepp, J. B. Ali, S. Ward, A. J. Green, J. R. Yates, J. Kwiatkowska, E. P. Henske, M. P. Short, J. H. Haines, S. Jozwiak and D. J. Kwiatkowski, "Identification of the Tuberous Sclerosis Gene TSC1 on Chromosome 9q34," Science, Vol. 277, No. 5327, August 1997, pp. 805-808. doi:10.1126/science. 277.5327 .805

[26] European Chromosome 16 Tuberous Sclerosis Consortium, "Identification and Characterization of the Tuberous Sclerosis Gene on Chromosome 16," Cell, Vol. 75, No. 7, December 1993, pp. 1305-1315. doi:10.1016/0092-8674(93)90618-Z

[27] G. Martignoni and M. Amin, “Angiomyolipoma,” In: J. N. Eble, G. Sauter, J. Epstein and I. Sesterhenn (eds), Pathology and Genetics of Tumours of the Urinary System and Male Genital Organs Series: WHO Classification of Tumours, IARC Press, Lyon, 2004, pp. 65-67. 